\title{
Mediative Fuzzy Extension Technique and Its Consistent Measurement in the Decision Making of Medical Application
}

\author{
M. K. Sharma $\mathbb{D D}^{1}{ }^{1}$ Nitesh Dhiman, ${ }^{1}$ Lakshmi Narayan Mishra $\mathbb{D}^{2}{ }^{2}$ \\ Vishnu Narayan Mishra ${ }^{(D)}{ }^{3}$ and Suresh Kumar Sahani ${ }^{4,5}$ \\ ${ }^{1}$ Department of Mathematics, C.C.S. University, Meerut-250004, India \\ ${ }^{2}$ Department of Mathematics, School of Advanced Sciences, Vellore Institute of Technology (VIT) University, Vellore 632014, \\ Tamil Nadu, India \\ ${ }^{3}$ Department of Mathematics, Indira Gandhi National Tribal University, Lalpur, Amarkantak, Anuppur, \\ Madhya Pradesh 484 887, India \\ ${ }^{4}$ Department of Mathematics, MIT Campus, T.U, Janakpurdham, Nepal \\ ${ }^{5}$ Department of Mathematics, Rajarshi Janak Campus, T.U, Janakpurdham 45600, Nepal
}

Correspondence should be addressed to Lakshmi Narayan Mishra; lakshminarayanmishra04@gmail.com and Vishnu Narayan Mishra; vishnunarayanmishra@gmail.com

Received 7 February 2021; Revised 16 March 2021; Accepted 8 April 2021; Published 28 April 2021

Academic Editor: G. Muhiuddin

Copyright (C) 2021 M. K. Sharma et al. This is an open access article distributed under the Creative Commons Attribution License, which permits unrestricted use, distribution, and reproduction in any medium, provided the original work is properly cited.

\begin{abstract}
The notion of fuzzy set theory has not so far been districted over medical diagnosis. There are some added applications, for example, in image processing, pattern identification, and many medical devices. In this research article, we introduced a new mediative fuzzy ranking technique as the fuzzy extension in decision making. The proposed mediative fuzzy logic-based technique is more relevant and applicable to incomplete and doubtful situations or some contradictions present in the expert knowledge. The value of the contradictory degree for mediative fuzzy sets used in the extension principle is defined. The proposed mediative fuzzy ranking method is easily implemented in the medical field, and the proposed mediative fuzzy extension-based measured technique is useful to medical experts and doctors in many decision-making situations; the entire work is illustrated with numerical examples. We have also given some future aspects of mediative fuzzy extension in the interpretation of type- 2 intuitionistic fuzzy sets.
\end{abstract}

\section{Introduction}

The successful list of applications of fuzzy set theory [1] has been rising quickly. Since 1965, fuzzy set theory has played a vital part in the development of many areas of mathematics, engineering, and science. In the medical field, uncertainty exists in the process of disease diagnosis and medical knowledge concerned with the relationship between symptoms and disease. This relationship constitutes a huge amount of impreciseness and vagueness in diagnostic procedures. In medical diagnosis, the doctor generally depends upon the knowledge based on what he/she gathers from the patient's past history, physician examination, test reports, and medical images such as $\mathrm{CT}$ scan and X-rays. Each of these sources contains a large number of uncertainties. In order to face uncertainty, the physician knowledge is represented by fuzzy relationship [2] between symptoms and disease. This fuzzy relation relates to the compositional fuzzy rule of inference in which each symptom is observed in the patient and to the set of various diseases. Adlassnig and Kolarz [3] elaborated this relational program to design a computerized system CADIAG-2 for medical diagnostic assistance. The approach of such a model is based on two types of relationships that presents tuck between symptoms and disease: occurrence and conformability relationship. Occurrence relation provided knowledge about the tendency of occurrence of symptoms when certain specific diseases are present, and the subsequent relation illustrates the power of symptoms toward validation of the existence of sickness. Thus, the CADIAG-2 system 
does not incorporate the relationship between symptoms and disease, but also between disease and symptoms themselves. Fordon and Bezdek [4] used another technique to model medical diagnosis by using fuzzy cluster analysis, in which the symptoms were designed with a membership function to represent each symptom category. Esogbue and Elder $[5,6]$ have also illustrated some techniques to the medical diagnostic system. The fuzzy numbers are incomparable; for many fuzzy decision-making problems, many [7] methods have been suggested. The first method is based on the Hamming distance, and the second method is based on the $\alpha$-cut method; furthermore, the supplementary method is based on the extension principle of fuzzy sets. As described earlier, the fuzzy theory takes interest in the description of the membership grade, and the non-membership degree is just complementary to the membership grade. However, the negation of the linguistic term does not satisfy the negation of the logical term in a valid mode. These linguistic contradictions do not satisfy the logical contradiction. There may be a few kinds of uncertainty present when selecting the membership degree. Membership function can be any function like triangular, trapezoidal, Gaussian, and exponential. Due to the uncertainty, nonmembership degree is less than or equal to the complement of the membership degree. Atanassov [8] recommended an intuitionistic fuzzy set to cover up the uncertainties in an appropriate way than the fuzzy sets. In the present work, the summation of the membership and non-membership grade is less or equal to "one," and hence the uncertainty or "unawareness" arises defining the membership grade. Thus, as compared to the fuzzy set theory, intuitionistic theory considers three types of uncertainties-membership grade, non-membership grade, and hesitation grade. For the studies of many decision-making problems [9], various comparison techniques of fuzzy numbers have been given in $[10,11]$. Jana et al. [12] used the Dombi operations to construct "Q-rung ortho pair fuzzy Dombi aggregation operators" and used the concept to build a mathematical model to solve the "multiattribute decision-making problems."

The similarity measure technique has been widely used in many fields. Other study on similarity measure through interval-valued type-2 intuitionistic fuzzy set [13] has also been studied. This study shows the dual concept of some "semimetric distances" between various intuitionistic fuzzy sets.

However, due to the benefit of intuitionistic fuzzy set over the ordinary fuzzy set, intuitionistic fuzzy sets receive a little attention together with its corresponding measured techniques in $[14,15]$. The intuitionistic fuzzy sets and interval-valued intuitionistic fuzzy sets have also been applied in the "dynamic hybrid multiattribute decision making (DHMADM)" [16], in which the contribution of dynamic weighted aggregation operators has also been described. Also, the Dombi operations were used to create some Pythagorean fuzzy Dombi aggregation operators [17] and build a mathematical model to solve the multiattribute decisionmaking problem in Pythagorean environment. Later, the concept of Pythagorean fuzzy multisets [18] has been used in medical diagnostic process.
Later in [19], the authors used the concept of mediative fuzzy logic in the management of contradictory information and showed the capability of mediative fuzzy logic over the intuitionistic and traditional fuzzy logics. There are other applications of mediative fuzzy logic in medical field given in $[9,20]$. The basic objectives of the proposed approach are as follows:

(1) There are so many techniques that have been applied in the similarity measurement and ranking between two fuzzy sets or two intuitionistic fuzzy sets.

(2) The well-defined extension principle will also extend over mediative fuzzy sets.

(3) A compositional fuzzy relation between patients with symptoms and symptoms with disease has also been defined over meditative fuzzy sets.

(4) We will define a priority contradictory function for the ranking purpose of two mediative fuzzy sets. The proposed tool will be easy to calculate and can easily be applicable in contradictory situations.

In the present work, we initiated with a comprehensive technique for a mediative fuzzy ranking method based on extension principle for mediative fuzzy set to construct a crisp order from the mediative fuzzy sets. In this paper, we are also giving an alternative approach for the better enhancement of medical diagnosis by using the utility of mediative fuzzy ranking analysis based on extension principle. The work in this present research article is divided into eight sections. In Section 2, we tried to explain a set of mathematical terms for easy communication with mediative fuzzy set and the extension principle for mediative fuzzy sets. Section 3 represents a mediative fuzzy relation in medical knowledge as shown in Figure 1. In Section 4 of the research paper, we formulated the algorithm. Section 5 and Section 5.1 introduce an intuitionistic fuzzy ranking method using the intuitionistic fuzzy extension and mediative fuzzy ranking method using the mediative fuzzy extension principle. Section 6 and Section 6.1 explain the applications of the proposed technique based on intuitionistic and mediative intuitionistic fuzzy extension in medical diagnosis. Section 7 presents the future aspect and real-life applications of the proposed technique. In Section 8, we tried to sketch the skeleton of the conclusion.

\section{Basic Terminologies}

In this section, we present some basic terminologies and the definitions associated with mediative fuzzy sets and the extension of mediative fuzzy sets.

2.1. Intuitionistic Fuzzy Set. Consider $A \subseteq X$ where $X$ is a universal set [8]; then, $\left.A=\left\{\left(x, \mu_{A}(x), v_{A}(x)\right): \forall x \in X\right)\right\}$, where $\mu_{A}(x)$ and $v_{A}(x): X \longrightarrow[0,1]$ with $0 \leq \mu_{A}(x), v_{A}(x) \leq 1$ called the membership and non-membership grades of $x$ in Xrespectively and $\pi_{A}(x)=1-\mu_{A}(x)-v_{A}(x)$ called hesitation grade of $x$ in $X$. 

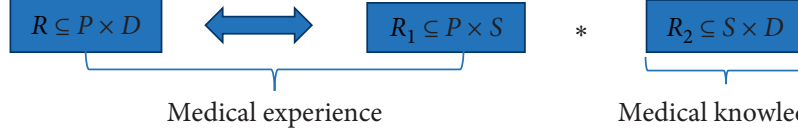

Medical knowledge

$R$ : Mediative fuzzy relation connecting patients $P$ with disease $D$;

$R_{1}$ : Mediative fuzzy relation on set of patients $P$ with symptoms $S$;

$R_{2}$ : Mediative fuzzy relation which represents the medical awareness

to relate the symptoms $S$.

Figure 1: Mediative fuzzy relation involved in medical diagnostic system.

2.2. Intuitionistic Fuzzy Number. An intuitionistic fuzzy $\operatorname{set} A$ is said to be an intuitionistic fuzzy number [21] if it satisfies the following axioms:

(i) $A$ is "normal," i.e., $\exists$ at least one $t \in X$ such that $\mu_{A}(t)=1$. Hence, $v_{A}(t)=0$.

(ii) Ais "convex," i.e., $\forall t_{1}, t_{2} \in X$ and $\gamma \in[0,1]$, such $\mu_{A}\left(\gamma t_{1}+(1-\gamma) t_{2}\right) \geq \min \left(\mu_{A}\left(t_{1}\right), \mu_{A}\left(t_{2}\right)\right)$, and that $v_{A}\left(\gamma t_{1}+(1-\gamma) t_{2}\right) \leq \max \left(v_{A}\left(t_{1}\right), v_{A}\left(t_{2}\right)\right)$.

(iii) Both $\mu_{A}(X), v_{A}(X)$ are piece-wise continuous.

2.3. Extension for Intuitionistic Fuzzy Sets. Let $X=A_{1} * A_{2} *, \ldots \ldots, A_{r}$, where $A_{i}, i=1,2, \ldots \ldots, r$ are intuitionistic fuzzy sets, and let $Y$ be another set. Let $\varnothing: X \longrightarrow Y$ such that

$$
\begin{aligned}
y & =\varnothing(x)=\varnothing\left(x_{1}, x_{2}, \ldots, x_{r}\right) ; \\
x_{i} & \in A_{i}, \\
x & =\left(x_{1}, x_{2}, \ldots, x_{r}\right) \in X .
\end{aligned}
$$

Then, the extension $\varnothing$ defines another intuitionistic fuzzy set in $Y$ as

$$
B=\left\{\left(y, \mu_{B}(y), v_{B}(y): \varnothing(x)=y \text { and } x \in X\right)\right\},
$$

where

$$
\begin{aligned}
& \mu_{B}(y)= \begin{cases}\operatorname{Sup}_{x \in \varnothing^{-1}(y)} \min _{i=1,2, \ldots, r}\left(\mu_{A_{i}}(x)\right), & \text { if } \varnothing^{-1}(y) \neq \varnothing, \\
0, & \text { else, }\end{cases} \\
& v_{B}(y)= \begin{cases}\operatorname{Inf}_{x \in \varnothing^{-1}(y)} \max _{i=1,2, \ldots, r}\left(\nu_{A_{i}}(x)\right), & \text { if } \varnothing^{-1}(y) \neq \varnothing \\
1, & \text { else, }\end{cases}
\end{aligned}
$$

where $\varnothing^{-1}(y)$ is the inverse image of $y$.

2.4. Mediative Fuzzy Set. The mediative fuzzy set [9] is the set of such triplets over a given universal set which contains membership function, non-membership function, and index of uncertainty with contradictory function [9].

Let $X$ be a universal set and $M \subseteq \mathrm{X}$; then, a mediative fuzzy set Min $X$ is defined as

$$
M=\left\{\frac{\left(x, \mu_{M}(x), v_{M}(x)\right)}{x} \in X\right\}
$$

where

$$
\left.\zeta_{M}(x)=\min \left(\mu_{M}(x), v_{M}(x)\right)\right)
$$

is called the "contradictory factor" of Min $X$ and

$$
\mu_{M}(x): X \longrightarrow[0,1], \nu_{M}(x): X \longrightarrow[0,1]
$$

with

$$
\begin{gathered}
\left(\gamma_{M}(x)+\frac{\zeta_{M}(x)}{2}\right)=1-\mu_{M}(x)-\nu_{M}(x), \\
0 \leq\left(\gamma_{M}(x)+\frac{\zeta_{M}(x)}{2}\right) \leq 1 \text { with }-0.25 \leq \gamma_{M} \leq 1 .
\end{gathered}
$$

Then, $M$ is called mediative fuzzy set defined on $X$.

$\mu_{M}(x)$ and $\nu_{M}(x)$ called the degree of membership and non-membership function respectively and $\gamma_{M}(x)+\left(\zeta_{M}(x) / 2\right)$ called the "index of uncertainty" with contradiction about " $\mathrm{x}$," if $\gamma_{M}(x)+\left(\zeta_{M}(x) / 2\right)$ is less then we know more about " $\mathrm{x}$," whether $\mathrm{x}$ belongs or not belongs to X. If $\gamma_{M}(x)+\left(\zeta_{M}(x) / 2\right)$ has value large then we know less about " $\mathrm{x}$," and if $\gamma_{M}(x)+\left(\zeta_{M}(x) / 2\right)=0$, then mediative fuzzy set coverts to traditional fuzzy set, and if $\mu_{M}(x)$ and $1-v_{M}(x)$ both are equals to 0 or 1 , then it will convert into bi-valued logic.

2.5. Extension for Mediative Fuzzy Sets. Let $X=A_{1} * A_{2} *, \ldots \ldots, A_{r}$ where $A_{i}, i=1,2, \ldots \ldots, r$ are the mediative fuzzy sets and let $Y$ be another set. Let $\varnothing: X \longrightarrow Y$ such that $y=\varnothing(x)=\varnothing\left(x_{1}, \quad x_{2}, \ldots \ldots\right.$, $\left.x_{r}\right) ; x_{i} \in A_{i}$ and $x=\left(x_{1}, x_{2}, \ldots \ldots, x_{r}\right) \in X$. Then, the extension $\varnothing$ defines another mediative fuzzy set $B$ in $Y$ as

$$
B=\left\{\left(y, \mu_{B}(y), v_{B}(y): \varnothing(x)=y \text { and } x \in X\right)\right\},
$$

where

$$
\begin{aligned}
& \mu_{B}(y)= \begin{cases}\operatorname{Sup}_{x \in \varnothing^{-1}(y)} \min _{i=1,2, \ldots, r}\left(\mu_{A_{i}}(x)\right), & \text { if } \varnothing^{-1}(y) \neq \varnothing, \\
0, & \text { else, }\end{cases} \\
& v_{B}(y)= \begin{cases}\operatorname{Inf}_{x \in \varnothing^{-1}(y)} \max _{i=1,2, \ldots, r}\left(v_{A_{i}}(x)\right), & \text { if } \varnothing^{-1}(y) \neq \varnothing, \\
1, & \text { else, }\end{cases}
\end{aligned}
$$

where $\varnothing^{-1}(y)$ is the inverse image of $y$.

2.6. Contradictory Function Value. The value of contradictory function can be calculated by

$$
\xi_{B}(y)=\min \left(\mu_{B}(y), v_{B}(y)\right) .
$$




\section{Mediative Fuzzy Relation in Medical Knowledge}

For the purpose of our study, we have given a compositional rule for the intuitionistic fuzzy relationship to the diagnostic process as follows:

$R$ : mediative fuzzy relation connecting patients $P$ with disease $D$.

$R_{1}$ : mediative fuzzy relation on set of patients $P$ with symptoms $S$.

$R_{2}$ : mediative fuzzy relation that represents the medical awareness to relate the symptoms $S$ to diseases and diseases $D$.

\section{Algorithm}

The algorithm is divided into two parts: part (A) presents the algorithm for intuitionistic fuzzy set which depends upon the membership and non-membership values and part (B) presents the algorithm for mediative fuzzy sets, which is based upon a single contradictory function value.

\subsection{For Intuitionistic Fuzzy Sets.}

Step 1: let us consider two intuitionistic fuzzy sets $A\left(=A_{i}\right)$ and $B\left(=B_{i}\right)$ and take their membership and non-membership values.

Step 2: calculate a "priority" function $P$ on the ordered pair $(A, B)$ based on their membership and nonmembership values.

Step 3: in step 3, we have the following conclusions:

(i) $\operatorname{If} P(A) \leq P(B) \Rightarrow A \leq B$.

(ii) If $P(A) \geq P(B) \Rightarrow A \geq B$.

4.2. For Mediative Fuzzy Sets.

Step 1: let us consider two mediative fuzzy sets $A\left(=A_{i}\right)$ and $B\left(=B_{i}\right)$ and find their contradictory values.

Step 2: find the "priority" contradictory function $P$ on the ordered pair $(A, B)$.

Step 3: after step 2, we have the following conclusions:

(i) If $P(A) \leq P(B) \Rightarrow A \leq B$ (based on contradictory values).

(ii) If $P(A) \geq P(B) \Rightarrow A \geq B$.

\section{Ranking Method Based on Extension of Intuitionistic Fuzzy Sets}

The intuitionistic fuzzy numbers are not comparable. In any intuitionistic fuzzy decision making, we need a comprehensive technique. We adopt an intuitionistic fuzzy ranking method based on extension principle to construct a crisp ordering from intuitionistic fuzzy numbers. In this section, we construct an intuitionistic fuzzy set $P$ for intuitionistic fuzzy numbers called "priority." Let us consider two intuitionistic fuzzy sets $A\left(=A_{i}\right)$ and $B\left(=B_{i}\right)$ and then define a "priority" intuitionistic fuzzy set $P$ on the ordered pair $(A, B)$ as follows:

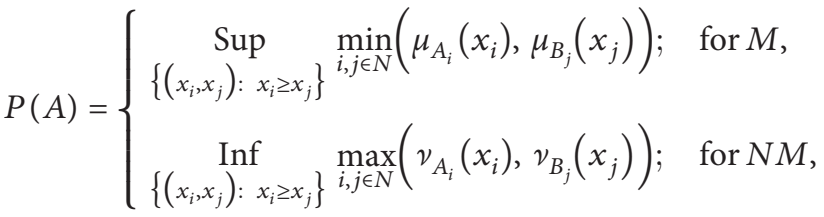

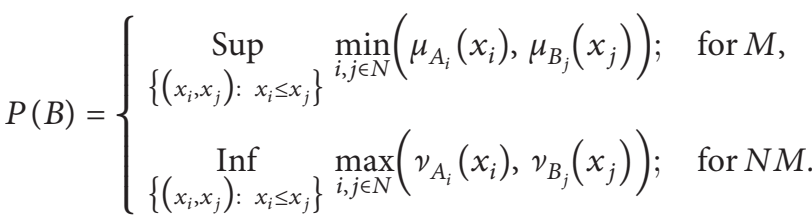

If $P(A) \leq P(B)$ (for membership/M) and

$$
P(A) \geq P(B)\left(\frac{\text { for non }- \text { membership }}{N M}\right) \Rightarrow A \leq B .
$$

5.1. Extension of Mediative Fuzzy Sets. The mediative fuzzy sets are not comparable. In any mediative fuzzy decision making, we need a comprehensive technique. We adopt a mediative fuzzy ranking method based on extension principle to construct a crisp ordering from mediative fuzzy sets. In this section, we construct a mediative fuzzy set $P$ for mediative fuzzy sets called "priority." Let us consider two mediative fuzzy sets $A\left(=A_{i}\right)$ and $B\left(=B_{i}\right)$ and then define a "priority" contradictory function $P$ on the ordered pair $(A, B)$ as follows:

$$
\begin{gathered}
P(A)=\operatorname{Sup}_{\left\{\left(x_{i}, x_{j}\right): x_{i} \geq x_{j} / M\right\}} \min _{i, j \in N}\left(\frac{1}{2} \xi_{A_{i}}\left(x_{i}\right), \frac{1}{2} \xi_{B_{j}}\left(x_{j}\right)\right), \\
P(B)=\operatorname{Sup}_{\left\{\left(x_{i}, x_{j}\right): x_{i} \leq x_{j} / M\right\}} \min _{i, j \in N}\left(\frac{1}{2} \xi_{A_{i}}\left(x_{i}\right), \frac{1}{2} \xi_{B_{j}}\left(x_{j}\right)\right) .
\end{gathered}
$$
grade.

\section{Applications Based on Extension of Intuitionistic Fuzzy Sets in Medical Diagnosis}

Consider a patient $p$ who has four symptoms represented by $S=\left\{s_{1}, s_{2}, s_{3}, s_{4}\right\}$ and their severity level is defined by the intuitionistic fuzzy set:

$$
I_{p}=\sum_{i=1}^{4} \frac{I_{p}\left(s_{i}\right)}{s_{i}}=\frac{(0.1,0.4)}{s_{1}}+\frac{(0.3,0.7)}{s_{2}}+\frac{(0.4,0.6)}{s_{3}}+\frac{(0.6,0.4)}{s_{4}},
$$

where $I_{p}\left(s_{i}\right) \in[0,1], i=1,2,3,4$ denotes the degree of membership and non-membership in the intuitionistic fuzzy set describing the patient $p$ and is prescribed on the set $S$, which points out the level of severity of the symptom $s_{i}, i=1$, 2, 3, 4 for the patient. Now, our objective is to determine the proper diagnosis for this patient out of the three possible diseases $D=\left\{d_{1}, d_{2}, d_{3}\right\}$. Each of these diseases is described 
by an intuitionistic fuzzy set, which gives the ordinary range of severity of respective symptom that may be familiar with the patient with the respective disease. So, the set of disease $D$ is described by the following sets:

$$
\begin{aligned}
& I_{d_{j}}=\sum_{i=1}^{4} \frac{I_{d_{j}}\left(s_{i}\right)}{s_{i}}, \quad j=1,2,3, \\
& I_{d_{1}}=\frac{(0.2,0.3)}{s_{1}}+\frac{(0.2,0.8)}{s_{2}}+\frac{(0.6,0.4)}{s_{3}}+\frac{(0.1,0.9)}{s_{4}}, \\
& I_{d_{2}}=\frac{(0,1)}{s_{1}}+\frac{(1,0)}{s_{2}}+\frac{(0.5,0.4)}{s_{3}}+\frac{(0.3,0.6)}{s_{4}} \\
& I_{d_{3}}=\frac{(0.1,0.8)}{s_{1}}+\frac{(0,1)}{s_{2}}+\frac{(0.8,0.2)}{s_{3}}+\frac{(0,1)}{s_{4}} .
\end{aligned}
$$

For each $j=1,2,3, I_{d_{j}}$ denotes the intuitionistic fuzzy set where $I_{d_{j}}\left(s_{i}\right)$ represents the membership and nonmembership degree of the symptom $s_{i}$ for the disease $d_{j}, j=1,2,3$. We further define an intuitionistic fuzzy relation $R$ to represent the medical judgment that connects the set of symptoms $S$ with their respective disease from $D$ that particularizes the efficiency of various symptoms $s_{i}$ for the diagnosis of the respective diseased $d_{j}$. Further, to the relevancy of respective symptom $s_{i}$ with respective disease $d_{j}$ defining an intuitionistic fuzzy relation $R=R\left(s_{i}, d_{j}\right)$ by weights matrix as;

$$
R\left(s_{i}, d_{j}\right)=\begin{gathered}
d_{1} \\
s_{1} \\
s_{2} \\
s_{3} \\
s_{4}
\end{gathered}\left[\begin{array}{ccc}
0.3 & 0.8 & d_{2} \\
0.6 & 0.5 & 0.3 \\
0.7 & 0.2 & 0.9 \\
0.8 & 0.6 & 0.3
\end{array}\right]
$$

where the matrix $R\left(s_{i}, d_{j}\right)=S * D$ represents the weight of each symptom $s_{i}$ with their respective disease $d_{j}$. For the diagnosis of the patient $p$, we use a clustering-based technique to find out that which diagnostic cluster as given in $I_{d_{j}}$, the patient is uttermost identical. Present technique is executed by enumerate a closeness measure between the symptoms $s_{i}$ and diseases $d_{j}$ To figure out this similarity, we use an intuitionistic fuzzy ranking method using intuitionistic fuzzy extension.

$$
\begin{gathered}
P\left(I_{p}\right)=\left\{\begin{array}{cl}
\operatorname{Sup}_{\left\{\left(s_{i}, s_{k}\right): s_{i} \geq s_{k}\right\}} \min _{i, k \in N}\left(\mu_{I_{p}}\left(s_{i}\right), \mu_{I_{d_{j}}}\left(s_{k}\right)\right) ; & \text { for } M, \\
\operatorname{Inf}_{\left\{\left(s_{i}, s_{k}\right): s_{i} \geq s_{k}\right\}} \max _{i, k \in N}\left(\nu_{I_{p}}\left(s_{i}\right), \nu_{I_{d_{j}}}\left(s_{k}\right)\right) ; & \text { for } N M,
\end{array}\right. \\
P\left(I_{d_{j}}\right)=\left\{\begin{array}{cl}
\operatorname{Sup}_{\left\{\left(s_{i}, s_{k}\right): s_{i} \leq s_{k}\right\}} \min _{i, k \in N}\left(\mu_{I_{p}}\left(s_{i}\right), \mu_{I_{d_{j}}}\left(s_{k}\right)\right) ; & \text { for } M, \\
\operatorname{Inf}_{\left\{\left(s_{i}, s_{k}\right): s_{i} \leq s_{k}\right\}} \max _{i, k \in N}\left(\nu_{I_{p}}\left(s_{i}\right), \nu_{I_{d_{j}}}\left(s_{k}\right)\right) ; & \text { for } N M .
\end{array}\right.
\end{gathered}
$$

$$
\begin{aligned}
& I_{p}=\frac{(0.1,0.4)}{s_{1}}+\frac{(0.3,0.7)}{s_{2}}+\frac{(0.4,0.6)}{s_{3}}+\frac{(0.6,0.4)}{s_{4}}, \\
& I_{d_{1}}=\frac{(0.2,0.3)}{s_{1}}+\frac{(0.2,0.8)}{s_{2}}+\frac{(0.6,0.4)}{s_{3}}+\frac{(0.1,0.9)}{s_{4}} \text {, }
\end{aligned}
$$

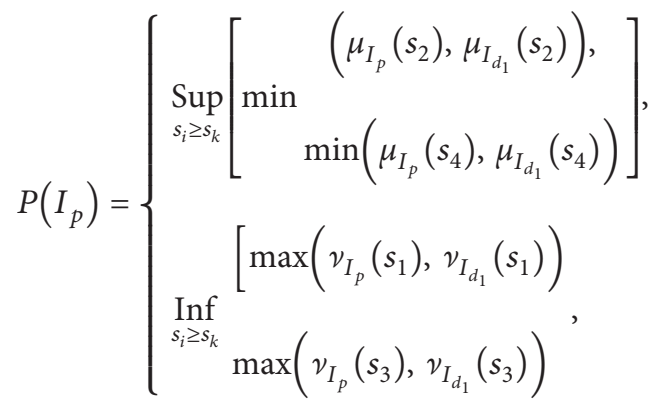

$$
\begin{aligned}
& P\left(I_{d_{1}}\right)=\left\{\begin{array}{c}
\min \left(\mu_{I_{p}}\left(s_{1}\right), \mu_{I_{d_{1}}}\left(s_{1}\right)\right), \\
\operatorname{Sup}_{s_{i} \leq s_{k}} \min \left(\mu_{I_{p}}\left(s_{3}\right), \mu_{I_{d_{1}}}\left(s_{3}\right)\right) \\
\operatorname{Inf}_{s_{i} \leq s_{k}}\left[\begin{array}{c}
\left(v_{I_{p}}\left(s_{2}\right), v_{I_{d_{1}}}\left(s_{2}\right)\right) \\
\max \left(v_{I_{p}}\left(s_{4}\right), v_{I_{d_{1}}}\left(s_{4}\right)\right)
\end{array}\right],
\end{array}\right. \\
& P\left(I_{p}\right)=\left\{\begin{array}{l}
0.6 \\
0.4
\end{array}\right. \\
& P\left(I_{d_{1}}\right)=\left\{\begin{array}{l}
0.4, \\
0.8,
\end{array}\right.
\end{aligned}
$$

For $d_{2}$ disease, $P\left(I_{p}\right)=\left\{\begin{array}{l}0.3 \\ 0.6\end{array}, P\left(I_{d_{2}}\right)=\left\{\begin{array}{l}0.4 \\ 0.6\end{array}\right.\right.$. For $d_{3}$ disease, $P\left(I_{p}\right)=\left\{\begin{array}{l}0.1 \\ 0.6\end{array}, P\left(I_{d_{3}}\right)=\left\{\begin{array}{l}0.4 \\ 0.8\end{array}\right.\right.$.

We may conclude that, according to the first priority, we have $P\left(I_{p}\right) \leq P\left(I_{d_{1}}\right)$. When we apply the same method over $d_{2}$, then the priority set gives $P\left(I_{p}\right) \geq P\left(I_{d_{2}}\right)$ and for $d_{3}$, we have $P\left(I_{p}\right) \geq P\left(I_{d_{3}}\right)$. The most likely disease from this measured technique attains the maximum value. Results in the symptoms of patient $p$ are uttermost similar to the disease $d_{1}$.

6.1. Extension of Mediative Fuzzy Sets in Medical Diagnosis. Consider a patient $p$ who has four symptoms represented by $S=\left\{s_{1}, s_{2}, s_{3}, s_{4}\right\}$ and their severity level is defined by the mediative fuzzy set:

$I_{p}=\sum_{i=1}^{4} \frac{I_{p}\left(s_{i}\right)}{s_{i}}=\frac{(0.1,0.4)}{s_{1}}+\frac{(0.3,0.7)}{s_{2}}+\frac{(0.4,0.6)}{s_{3}}+\frac{(0.6,0.4)}{s_{4}}$,

$\operatorname{Ford}_{1}$, 
where $I_{p}\left(s_{i}\right) \in[0,1], i=1,2,3,4$ denotes the degree of membership and non-membership in the mediative fuzzy set describing the patient $p$ with some contradictory factor and is prescribed on the set $S$, which points out the level of severity of the symptom $s_{i}, i=1,2,3,4$ for the patient. Now, our objective is to determine the proper diagnosis for this patient out of the three possible diseases $D=\left\{d_{1}, d_{2}, d_{3}\right\}$. Each of these diseases is described by an intuitionistic fuzzy set, which gives the ordinary range of severity of respective symptom that may be familiar with the patient with the respective disease. So, the set of disease $D$ is described by the following sets:

$$
\begin{aligned}
& I_{d_{j}}=\sum_{i=1}^{4} \frac{I_{d_{j}}\left(s_{i}\right)}{s_{i}}, \quad j=1,2,3, \\
& I_{d_{1}}=\frac{(0.2,0.3)}{s_{1}}+\frac{(0.2,0.8)}{s_{2}}+\frac{(0.6,0.4)}{s_{3}}+\frac{(0.1,0.9)}{s_{4}}, \\
& I_{d_{2}}=\frac{(0,1)}{s_{1}}+\frac{(1,0)}{s_{2}}+\frac{(0.5,0.2)}{s_{3}}+\frac{(0.3,0.6)}{s_{4}} \\
& I_{d_{3}}=\frac{(0.1,0.8)}{s_{1}}+\frac{(0,1)}{s_{2}}+\frac{(0.8,0.2)}{s_{3}}+\frac{(0,1)}{s_{4}}
\end{aligned}
$$

For each $j=1,2,3, I_{d}$ denotes the mediative fuzzy set where $I_{d_{j}}\left(s_{i}\right)$ represents the membership and non-membership degree of the symptom $s_{i}$ for the disease $d_{j}, j=1,2,3$. We further define a mediative fuzzy relation $R$ to represent the medical judgment that connects the set of symptoms $S$ with their respective disease from $D$ that particularizes the efficiency of various symptoms $s s_{i}$ for the diagnosis of the respective disease $d_{j}$. Further, to the relevancy of respective symptom $s_{i}$ with respective disease $d_{j}$ defining a mediative fuzzy relation $R=R\left(s_{i}, d_{j}\right)$ by weights matrix as;

$$
\begin{aligned}
& \begin{array}{lll}
d_{1} & d_{2} & d_{3},
\end{array} \\
& R\left(s_{i}, d_{j}\right)=s_{2}\left[\begin{array}{ccc}
0.3 & 0.8 & 1 \\
s_{3} & s_{3} \\
s_{4} & 0.6 & 0.3 \\
0.7 & 0.2 & 0.9 \\
0.8 & 0.6 & 0.3
\end{array}\right],
\end{aligned}
$$

where the matrix $R\left(s_{i}, d_{j}\right)=S * D$ represents the weight of each symptom $s_{i}$ with their respective disease $d_{j}$. For the diagnosis of the patient $p$, we use a clustering based technique to find out that which diagnostic cluster as given in $I_{d_{j}}$, the patient is uttermost identical. Present technique is executed by enumerate a closeness measure between the symptomss $s_{i}$ and disease $d_{j}$. To figure out this similarity, we use a mediative fuzzy ranking method using mediative fuzzy extension.

$$
\begin{gathered}
P\left(I_{p}\right)=\operatorname{Sup}_{\left\{\left(s_{i}, s_{k}\right): s_{i} \geq s_{k}\right\} / M} \min _{i, k \in N}\left(\frac{1}{2} \xi_{I_{p}}\left(s_{i}\right), \frac{1}{2} \xi_{I_{d_{j}}}\left(s_{k}\right)\right), \\
P\left(I_{d_{j}}\right)=\operatorname{Sup}_{\left\{\left(s_{i}, s_{k}\right): s_{i} \leq s_{k}\right\} / M} \min _{i, k \in N}\left(\frac{1}{2} \xi_{I_{p}}\left(s_{i}\right), \frac{1}{2} \xi_{I_{d_{j}}}\left(s_{k}\right)\right) .
\end{gathered}
$$

For $d_{1}$

$$
\begin{aligned}
& I_{p}=\frac{(0.1,0.4)}{s_{1}}+\frac{(0.3,0.7)}{s_{2}}+\frac{(0.4,0.6)}{s_{3}}+\frac{(0.6,0.4)}{s_{4}}, \\
& I_{d_{1}}=\frac{(0.2,0.3)}{s_{1}}+\frac{(0.2,0.8)}{s_{2}}+\frac{(0.6,0.4)}{s_{3}}+\frac{(0.1,0.9)}{s_{4}} \text {, } \\
& P\left(I_{p}\right)=\operatorname{Sup}_{s_{i} \geq s_{k} / M}\left[\begin{array}{l}
\min \left(\frac{1}{2} \xi_{I_{p}}\left(s_{2}\right), \frac{1}{2} \xi_{I_{d_{1}}}\left(s_{2}\right)\right) \\
\min \left(\frac{1}{2} \xi_{I_{p}}\left(s_{4}\right), \frac{1}{2} \xi_{I_{d_{1}}}\left(s_{4}\right)\right)
\end{array}\right], \\
& \min \left(\frac{1}{2} \xi_{I_{p}}\left(s_{1}\right), \frac{1}{2} \xi_{I_{d_{1}}}\left(s_{1}\right)\right) \\
& P\left(I_{d_{1}}\right)=\operatorname{Sup}_{s_{i} \leq s_{k} / M} \\
& \min \left(\frac{1}{2} \xi_{I_{p}}\left(s_{3}\right), \frac{1}{2} \xi_{I_{d_{1}}}\left(s_{3}\right)\right)
\end{aligned}
$$

where $M$ stands for membership value, $P\left(I_{p}\right)=0.1$, $P\left(I_{d_{1}}\right)=0.2$; for $d_{2}$ disease, $P\left(I_{p}\right)=0.15, P\left(I_{d_{2}}\right)=0.1$; for disease $d_{3}, P\left(I_{p}\right)=0.15$ and $P\left(I_{d_{3}}\right)=0.1$ We may conclude that, according to the first priority mediative fuzzy set, we have $P\left(I_{p}\right) \leq P\left(I_{d_{1}}\right)$. When we apply the same method over $d_{2}$, then the priority mediative fuzzy set gives $P\left(I_{p}\right) \geq P\left(I_{d_{2}}\right)$ and for $d_{3}$, we have $P\left(I_{p}\right) \geq P\left(I_{d_{3}}\right)$. The most likely disease from this measured technique attains the maximum value. Results in the symptoms of patient $p$ are uttermost similar to the disease $d_{1}$.

\section{Future Aspect and Real-Life Applications}

We extend the idea of mediative fuzzy extension over type-2 intuitionistic fuzzy sets. In this, the membership/nonmembership functions of type-1 mediative fuzzy sets are crisp and the two-dimensional can be taken as in type-1 intuitionistic fuzzy set. So, type-1 intuitionistic fuzzy sets are sufficient for dealing with the uncertainty of the present model of this study. On the one hand, type- 2 intuitionistic fuzzy sets are capable of dealing with these uncertainties due to the three-dimensional nature of membership/nonmembership functions. So, mediative fuzzy set is not capable to instantly form the uncertainties present in any study. On the other hand, type-2 intuitionistic fuzzy sets have the capability to deal such type of uncertainties due to their membership/non-membership functions which are themselves fuzzy and three-dimensional. So, it gives the threedimensional degree that provides the added degree of freedom to handle such type of uncertainties. The arithmetic of type-2 intuitionistic fuzzy sets provided the clarity to deal the uncertainty, so everyone should believe in using the type2 intuitionistic fuzzy extension principle which is the generalization of Zadeh's [22] fuzzy extension. We have given a type-2 intuitionistic fuzzy set characterized by [23] type-2 membership/non-membership function $0 \leq \mu_{A}(x, u), \quad v_{A}$ $(x, u) \leq 1$ : 


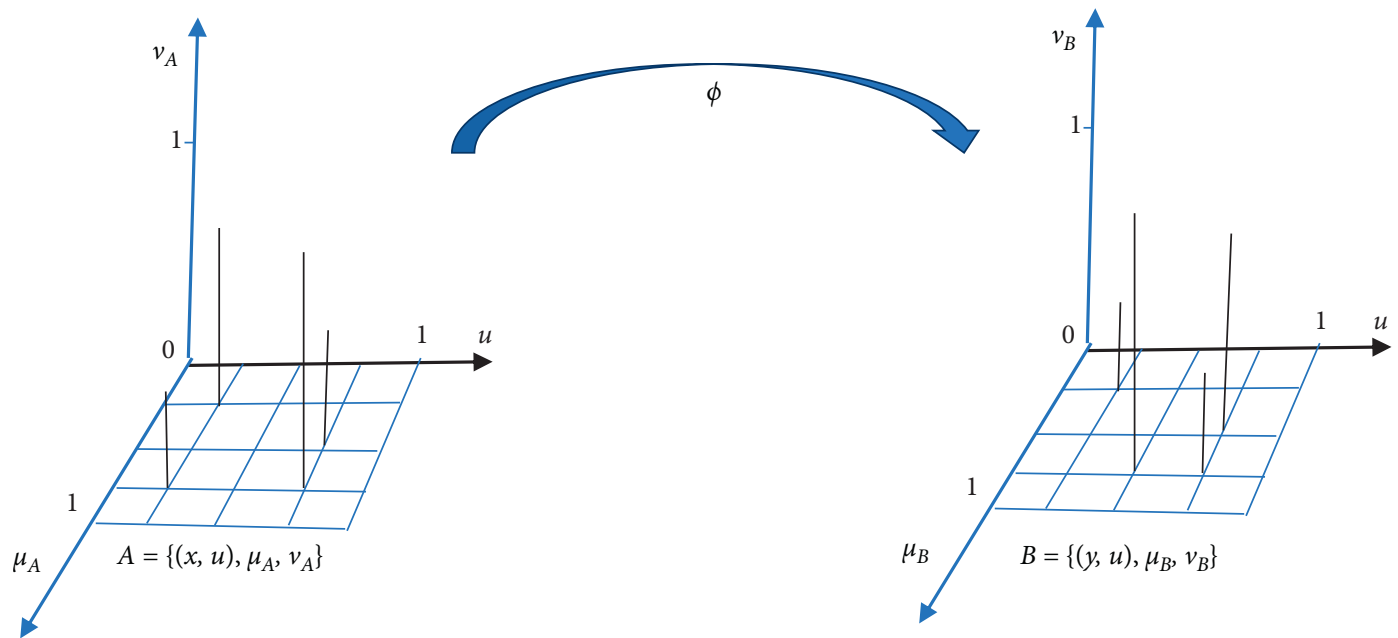

Figure 2: Geometrical representation of type-2 intuitionistic fuzzy extension.

TABLE 1: A comparative study among the existing approaches and the proposed mediative fuzzy logic-based measurement approach.

\begin{tabular}{|c|c|c|c|}
\hline Techniques & $\begin{array}{c}\text { Tools of } \\
\text { measurement }\end{array}$ & Computational results & Advantages of proposed approach \\
\hline Fuzzy logic approach & $\begin{array}{l}\text { Truth value based } \\
\text { only }\end{array}$ & $\begin{array}{l}\text { For } d_{1} \text { disease, } P\left(I_{p}\right)=0.6 \\
P\left(I_{d_{1}}\right)=0.4 \\
\text { For } d_{2} \text { disease, } P\left(I_{p}\right)=0.3 \\
P\left(I_{d_{2}}\right)=0.4 \\
\text { For } d_{3} \text { disease, } P\left(I_{p}\right)=0.1 \\
P\left(I_{d_{3}}\right)=0.4\end{array}$ & An ordinary solution, based only on membership value. \\
\hline \multirow{3}{*}{$\begin{array}{l}\text { Intuitionistic fuzzy } \\
\text { logic approach }\end{array}$} & \multirow{3}{*}{$\begin{array}{l}\text { Truth/false values } \\
\text { based }\end{array}$} & $\begin{array}{c}\text { For } d_{1} \text { disease, } P\left(I_{p}\right)=\left\{\begin{array}{l}0.6 \\
0.4\end{array}\right. \\
P\left(I_{d_{1}}\right)=\left\{\begin{array}{l}0.4 \\
0.8\end{array}\right.\end{array}$ & \multirow{3}{*}{$\begin{array}{l}\text { Better solution than fuzzy approach. But slightly tough to } \\
\text { understand and hard to calculate and time consuming. }\end{array}$} \\
\hline & & $\begin{array}{c}\text { For } d_{2} \text { disease, } P\left(I_{p}\right)=\left\{\begin{array}{l}0.3 \\
0.6\end{array}\right. \\
P\left(I_{d_{2}}\right)=\left\{\begin{array}{l}0.4 \\
0.6\end{array}\right.\end{array}$ & \\
\hline & & $\begin{array}{c}\text { For } d_{3} \text { disease, } P\left(I_{p}\right)=\left\{\begin{array}{l}0.1 \\
0.6\end{array}\right. \\
P\left(I_{d_{3}}\right)=\left\{\begin{array}{l}0.4 \\
0.8\end{array}\right.\end{array}$ & \\
\hline $\begin{array}{l}\text { Mediative fuzzy logic } \\
\text { approach }\end{array}$ & $\begin{array}{l}\text { Contradictory } \\
\text { value based }\end{array}$ & $\begin{array}{c}\text { For } d_{1} \text { disease, } P\left(I_{p}\right)=0.1 \\
P\left(I_{d_{1}}\right)=0.2 . \\
\text { For } d_{2} \text { disease, } P\left(I_{p}\right)=0.15 \\
P\left(I_{d_{2}}\right)=0.1 \\
\text { For disease } \\
d_{3}, P\left(I_{p}\right)=0.15 P\left(I_{d_{3}}\right)=0.1\end{array}$ & $\begin{array}{c}\text { Sigle value-based solution. Considering the membership, } \\
\text { non-membership, hesitation, and contradictory factors, it } \\
\text { is easy to compare and compute. Computes each and } \\
\text { every aspect of the model. }\end{array}$ \\
\hline
\end{tabular}

$A=\left\{\left(x, u, \mu_{A}(x, u), v_{A}(x, u)\right): \forall x \in X, \forall u \in I_{x} \subseteq[0,1)\right\}$

Let $B$ be another set defined on $Y$ and let $\phi: X \times I_{x} \longrightarrow Y \times I_{y}$ such that $(y, u)=\phi(x, u),(x, u) \epsilon$ $X \times I_{x}$; then, the extension $\phi$ defines a type-2 intuitionistic fuzzy set $B$ in $I_{y} \subseteq[0,1)$ as shown in Figure 2 , where $B=$ $\left.\left\{\left(y, u, \mu_{B}(y, u), v_{B}(y, u)\right): \quad(y, u)=\phi(x, u)\right)\right\}, \forall x \in X$. In decision making, type-2 intuitionistic fuzzy extension may play a vital role, and we may easily develop a comprehensive technique, by adopting a type-2 intuitionistic fuzzy ranking method based on extension principle to construct a crisp ordering for type-2 intuitionistic fuzzy sets. The type-2 intuitionistic fuzzy sets $A$ and $B$ can easily be compared by defining a priority function $P$ on $(A, B)$.

\section{Conclusion}

Since proper diagnosis is one of the most essential parts of decision making in the world of medical field, it is difficult to 
handle a diagnostic process when there is a contradiction present in the experts' knowledge. So, in order to carry out the diagnosis process, we have used the extension of fuzzy logic in the form of mediative logic, to improve the impure and uncertain values of parameter present in the medical field. In decision-making problems, meditative fuzzy sets are very fruitful in finding the shape of the outputs of the problem. In the consecutive decision-making problems, an ordering technique is required. Mediative fuzzy sets provide a best ordering technique that can be used in consecutive decision-making problems. Therefore, the whole work completed in this study enlightens the points as follows:

(1) The concept of extension principle over mediative fuzzy set has been introduced; furthermore, a mediative fuzzy ranking-based technique is also described that provides a well ordering of the mediative fuzzy sets.

(2) The given concept may also be used in decisionmaking for diagnosing the most likely same disease of this category. From the proposed measured technique, we can easily verify the patient symptoms most similar to a particular disease.

(3) In the application section of the research work, for a patient $p$, we have taken a set of diseases $D$ and a set of symptoms $S$ whose levels of severity are described in the form of a mediative fuzzy set. On the basis of their priority value, we can easily find the most likely disease from the measured technique. This research paper also illustrates the mediative relation between symptom and disease on the basis of a mediative fuzzy ranking technique based on extension principle.

(4) Furthermore, a new methodology for the interpretation of type-2 intuitionistic fuzzy set has also been discussed. Also, the given ranking method follows the popular existing extension principle based on a method that is described numerically.

(5) One major advantage of mediative fuzzy logic over intuitionistic fuzzy logic is that, during the comparison process of mediative fuzzy sets, we need only one single value of the contradictory function as an output, which gives an easiest tool to compare two mediative fuzzy sets. In general, to do work with an intuitionistic fuzzy set, we need to consider both the values during the ranking process, i.e., membership degree and non-membership degree, which is quite difficult and time-consuming process. So, the proposed method presents the utility of mediative fuzzy logic over intuitionistic fuzzy logic.

(6) A comparative study is also discussed in Table 1 between the existing approaches and the proposed approach, which shows the superiority of our proposed mediative fuzzy logic-based measurement approach.

On the basis of above, we may conclude that the present research paper describes the utility of mediative fuzzy logic in decision making. The uncertainties present in the membership degree and the non-membership degree of the mediative fuzzy set are described and their use in ranking technique has also been discussed. The given ranking method is effortlessly implemented and has a useful explanation over type-2 intuitionistic fuzzy sets.

\section{Data Availability}

We are sharing the source that includes the data that support the findings of our results and conclusion of the manuscript. The technique which we have given can be verified on the data which are available on the following hyperlinks: https:// www.mdpi.com/2073-8994/11/6/808 and https://www.icmr. gov.in/ (Micronutrient Profile of Indian Population by Indian Council of Medical Research).

\section{Conflicts of Interest}

The authors declare that they have no conflicts of interest.

\section{Acknowledgments}

The second author is grateful to the University Grant Commission (UGC) for economic assistance.

\section{References}

[1] L. A. Zadeh, "Fuzzy sets," Information and Control, vol. 8, no. 3, pp. 338-353, 1965.

[2] E. Sanchez, "Medical diagnosis and composite fuzzy relations," in Advances in Fuzzy Set Theory and Applications, M. M. Gupta, R. K. Ragade, and R. R. Yager, Eds., pp. 437444, North-Holland, New Work, NY, USA, 1979.

[3] K. P. Adlassnig and G. Kolarz, "CADIAG-2: computerassisted medical diagnosis using fuzzy subsets," in Approximate Reasioning in Decision Analysis, M. M. Gupta and Sanchez, Eds., pp. 219-247, North-Holland, New York, NY, USA, 1982.

[4] W. A. Fordon and J. C. Bezdek, "The application of fuzzy set theory to medical diagnosis," in Advances in Fuzzy Set Theory and Applications, M. M. Gupta, R. K. Ragede, and R. R. Yager, Eds., pp. 445-461, North-Holland, New York, NY, USA, 1979.

[5] A. O. Esogbue and R. C. Elder, "Fuzzy sets and the modelling of physician decision processes, part I: the initial interviewinformation gathering session," Fuzzy Sets and Systems, vol. 2, no. 4, pp. 279-291, 1979.

[6] A. O. Esogbue and R. C. Elder, "Fuzzy sets and the modelling of physician decision processes, part II: fuzzy diagnosis decision models," Fuzzy Sets and Systems, vol. 3, no. 1, pp. 1-9, 1980.

[7] G. J. Klir and B. Yuan, Fuzzy Sets and Fuzzy Logic: Theory and Applications, Pearson Education, London, UK, 2019.

[8] K. T. Atanassov, "Intuitionistic fuzzy sets," Fuzzy Sets and Systems, vol. 20, no. 1, pp. 87-96, 1986.

[9] N. Dhiman and M. K. Sharma, "Mediative multi-criteria decision support system for various alternatives based on fuzzy logic," International Journal of Recent Technology and Engineering (IJRTE), vol. 8, pp. 7940-7946, 2019.

[10] D. Dubois and H. Prade, "Ranking fuzzy numbers in the setting of possibility theory," Information Sciences, vol. 30, no. 3, pp. 183-224, 1983. 
[11] A. Taleshian and S. Rezvani, "Three-tier FMCDM problems with trapezoidal fuzzy number," World Applied Sciences Journal, vol. 10, pp. 1106-1113, 2010.

[12] C. Jana, G. Muhiuddin, and M. Pal, "Some Dombi aggregation of Q -rung orthopair fuzzy numbers in multiple-attribute decision making," International Journal of Intelligent Systems, vol. 34, no. 12, pp. 3220-3240, 2019.

[13] S. Cherif, N. Baklouti, H. Hagras, and A. M. Alimi, "Novel intuitionistic based interval type-2 fuzzy similarity measures with application to clustering," IEEE Transactions on Fuzzy Systems, 2021.

[14] D. F. Li, J. X. Nan, and M. J. Zhang, "A ranking method of triangular intuitionistic fuzzy numbers and application to decision making," International Journal of Computational Intelligence Systems, vol. 3, no. 5, pp. 522-530, 2010.

[15] H. B. Mitchell, "Ranking-intuitionistic fuzzy numbers," International Journal of Uncertainty, Fuzziness and KnowledgeBased Systems, vol. 12, no. 3, pp. 377-386, 2004.

[16] C. Jana and M. Pal, "A dynamical hybrid method to design decision making process based on GRA approach for multiple attributes problem," Engineering Applications of Artificial Intelligence, vol. 100, 2021.

[17] C. Jana, T. Senapati, and M. Pal, "Pythagorean fuzzy Dombi aggregation operators and its applications in multiple attribute decision-making," International Journal of Intelligent Systems, vol. 34, no. 9, pp. 2019-2038, 2019.

[18] E. P. Augustine, C. Jana, and M. pal, "Medical diagnostic process based on modified composite relation on Pythagorean fuzzy multi-sets," Granular Computing, pp. 1-9, 2021.

[19] O. Montiel, O. Castillo, P. Melin, and R. Sepulveda, "Mediative fuzzy logic: a new approach for contradictory knowledge management," Soft Computing, vol. 12, pp. 251-256, 2008.

[20] N. Dhiman and M. K. Sharma, "Mediative sugeno's-TSK fuzzy logic based screening analysis to diagnosis of heart disease," Applied Mathematics, vol. 10, no. 6, pp. 448-467, 2019.

[21] M. K. Sharma, N. Dhiman, and V. N. Mishra, "Mediative fuzzy logic of sugeno-tsk model for the diagnosis of diabetes," Journal of Physics: Conference Series, p. 1724, 2020.

[22] L. A. Zadeh, "The concept of a linguistic variable and its application to approximate reasoning-I," Information Sciences, vol. 8, no. 3, pp. 199-249, 1975.

[23] D. S. Surajit, M. B. Kar, S. Majumder, B. Roy, S. Kar, and D. Pamucar, "Intuitionistic type-2 fuzzy set and its properties," MDPI, vol. 11, p. 808, 2019. 